\title{
Experimental Studies on Joule Dissipation in a Nonequilibrium MHD Disk Generator
}

\author{
Student member \\ Hajime Nakamura \\ (Tokyo Institute of Technology), \\ Nonmember \\ Tetsuji Okamura \\ (Tokyo Institute of Technology), \\ Member \\ Susumu Shioda \\ (Tokyo Institute of Technology)
}

\section{Summary}

Joule dissipation in a nonequilibrium MHD disk generator was successfully estimated from power generation experiments. Faraday current in the disk generator was measured. The reduction of the total pressure caused by the Joule dissipation was also estimated. Experimental results suggest that the isentropic efficiency of the generator is strongly affected by the value of the Joule dissipation.

When the applied magnetic flux density increased, the extraction of electrical power increased remarkably, however the total pressure loss caused by the entropy production was suppressed. The high MHD interaction caused by the high magnetic flux density did not deteriorate the performance of the generator. It is considered from this fact that the application of higher magnetic flux density is essential to get higher isentropic efficiency.

Key words: MHD power generation, nonequilibrium disk generator, isentropic efficiency, Joule dissipation

\section{Introduction}

In the past several years, high enthalpy extraction from disk MHD generators has been demonstrated[1,2]. In the research work[3,4], it was reported that the enthalpy extraction ratio could be improved up to $32.4 \%$ (where thermal input is estimated based on zero $\mathrm{K}$ ), using helium as a working gas.

One of the next subjects on the disk generators is to improve the isentropic efficiency. There are two important factors for the entropy production in the nonequilibrium generators. One is a wall friction loss and the other is Joule dissipation.

The Joule dissipation in the generator was estimated from the measurements of Hall current, Faraday current and the estimation of electrical conductivity. Experiments were carried out varying operating conditions such as external load resistance, inlet stagnation temperature and applied magnetic flux density in order to investigate the effects of these operating conditions on the Joule dissipation.

\section{Experimental Apparatus}

The present disk generator was driven by a shock tube[5]. A schematic view of the disk channel is shown in Fig. 1. A set of 24 swirl vanes which form supersonic nozzles was located between $r=58.4 \mathrm{~mm}$ and $83.8 \mathrm{~mm}$. The swirl ratio $\left(u_{\theta} / u_{r}\right)$ at the exit of the swirl vanes was 1. The Mach number at the MHD channel inlet (outer side of anode, $r=100 \mathrm{~mm}$ ) was estimated to be 2.5 by a two dimensional calculation considering the boundary

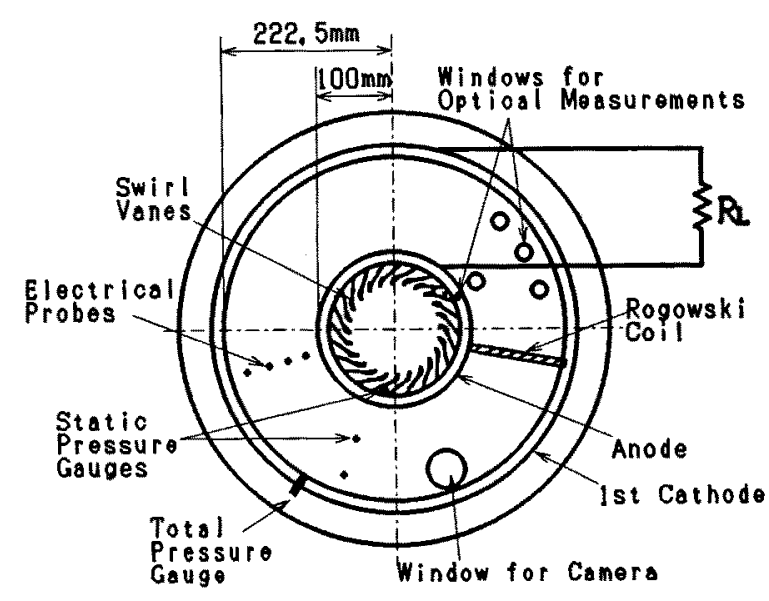

Figure 1 Schematic view of disk channel

layer.

A single external load resistance was connected between the anode and the 1st cathode in the present experiments to extract the electrical power.

The Faraday current, which flows in a circumferential direction in the generator, was measured using a Rogowski coil[6]. A Schematic view of the Rogowski coil is shown in Fig. 2. Measuring methods are described in Ref. [7]. The Rogowski coil was calibrated by supplying a known azimuthal current in the MHD channel before the power generation experiments. The effect of applied magnetic flux density on the output voltage of the Rogowski coil is small (smaller than 10\%). 


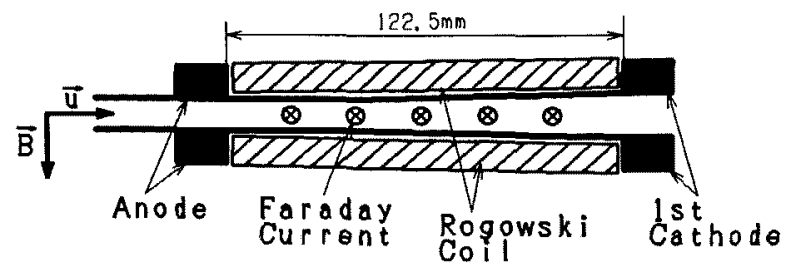

Figure 2 Schematic view of Rogowski coil

Table 1 Experimental conditions

\begin{tabular}{|c|c|}
\hline Working gas & He+Cs \\
\hline Stagnation temperature(K) & $1800 \sim 2400$ \\
Stagnation pressure(MPa) & $0.15 \sim 0.20$ \\
Seed fraction & $3 \sim 5 \times 10^{-4}$ \\
Thermal input $(\mathrm{MW})$ & $0.9 \sim 1.2$ \\
Load resistance $(\Omega)$ & $1.5 \sim 5$ \\
Magnetic flux density $(\mathrm{T})$ & $0.91 \sim 2.55$ \\
\hline
\end{tabular}

Total gas pressure at the exit of the channel (at the inner side of 1st cathode) was measured using a special type of Pitot tube which is hardly affected by the inclination of flow direction up to \pm 35 degrees[8]. Therefore, the measurements were performed with sufficient accuracy in spite of the flow swirl caused by inlet swirl vanes and Lorentz force.

\section{Experimental Results}

The experimental conditions used in the present study are listed in Table 1. Cesium seeded Helium was used as a working gas. Experiments were carried out varying the external load resistance $(1.5 \Omega \sim 5.0 \Omega)$, inlet stagnation temperature $(1800 K \sim 2400 K)$ and applied magnetic flux density $(0.91 T \sim 2.55 T)$.

〈3.1) Hall potential distributions The measured Hall potential distributions are shown in Fig. 3. A negative potential region appears at the generator inlet when the stagnation temperature is low or the magnetic flux density is low. This region is considered to be caused by the insufficient ionization in the supersonic nozzle. The Hall electric field becomes higher with the increase in the load resistance. When the stagnation temperature is high $(2400 K)$, a sudden deterioration of the Hall electric field appears at $r=120 \mathrm{~mm}$ although the Hall field is high at the inlet of the generator. This fact is thought to be caused by the deterioration of the electromotive force owing to a sudden reduction of the radial flow velocity and a reduction of Hall parameter, as suggested in Fig. 5 (b).

(3.2) Faraday current The variations of the measured Faraday current in the MHD channel are shown in Fig. 4. An increase in the Faraday current is found with the increase in the load resistance. This indicates that the Lorentz force against the flow direction becomes large when the load resistance is high. With the increase in stagnation temperature or magnetic flux density, there are no remarkable variations in the Faraday current. The tendency of the no increase in Faraday current with the increase in magnetic flux density indicates that the effective Hall parameter increases with the increase in the magnetic flux density.

$\langle 3 \cdot 3\rangle$ Static pressure distributions Static pressure distributions along the radial direction in the case of presence and absence of the applied magnetic field are shown in Fig. 5.

The static pressure rises in the generator with the increase in load resistance because the Lorentz force becomes larger due to the large Faraday current, as can be seen in Fig. 4.

When the stagnation temperature is high (2400K), the static pressure at $r=150 \mathrm{~mm}$ becomes high because of the existence of the strong Lorentz force at the generator inlet. When the stagnation temperature is low $(1800 K)$, the static pressure at $r=150 \mathrm{~mm}$ is higher than that of $2100 \mathrm{~K}$. It can be imagined from this fact that a steep rise in the static pressure in the upstream of $r=150 \mathrm{~mm}$ exists as shown in Fig. 5 (b) with a dotted line.

(3.4) Isentropic efficiency In the present experiments, isentropic efficiency $\eta_{i}$ is evaluated using the following equation,

$$
\eta_{i}=\frac{E \cdot E .}{1-\left(\frac{p_{0 \text { out }}}{p_{0 \text { in }}}\right)^{\frac{\gamma-1}{\gamma}}}
$$

where $E . E$. is enthalpy extraction ratio, $p_{0 \text { in }}$ and $p_{0 o u t}$ are inlet and outlet total gas pressures, respectively.

The variations of the enthalpy extraction ratio and the isentropic efficiency evaluated in the experiments are shown in Fig. 6. The maximum isentropic efficiency attained in the present experiments is $53 \%$ when the stagnation temperature is $2100 K$, as can be seen in Fig. 6 (b).

The value of the load resistance which gives maximum isentropic efficiency is $2.5 \Omega$ in the present experimental conditions. The lower values of the load resistance than this optimum value result in a steep decrease in the enthalpy extraction ratio and the isentropic efficiency. The higher values of it result in a gradual decrease in the enthalpy extraction ratio, however there is a steep decrease in the isentropic efficiency.

When the stagnation temperature is high $(2400 \mathrm{~K})$, the isentropic efficiency becomes lower than the case of $2100 \mathrm{~K}$. This fact is thought to be caused by the excessively high MHD interaction.

Both the enthalpy extraction ratio and the isentropic efficiency become higher with the increase in the magnetic flux density. A tendency of saturation is shown in the case of high magnetic flux density. This is caused by the high MHD interaction. 


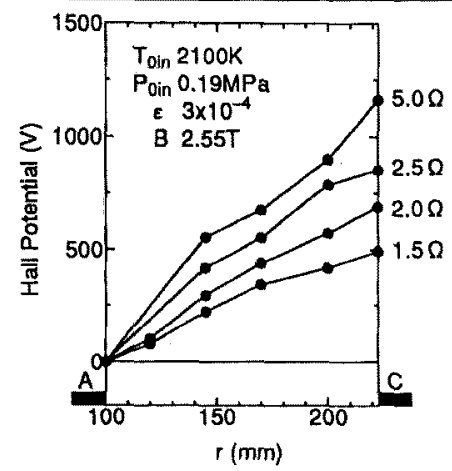

(a) vs. external load resistance

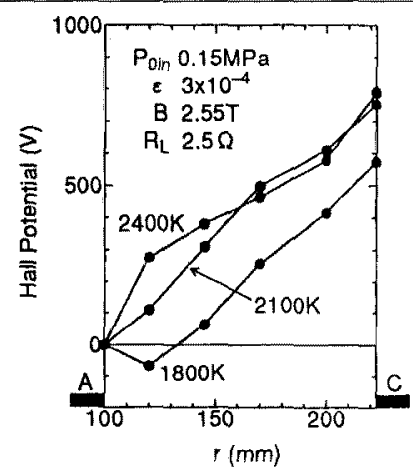

(b) vs. stagnation gas temperature

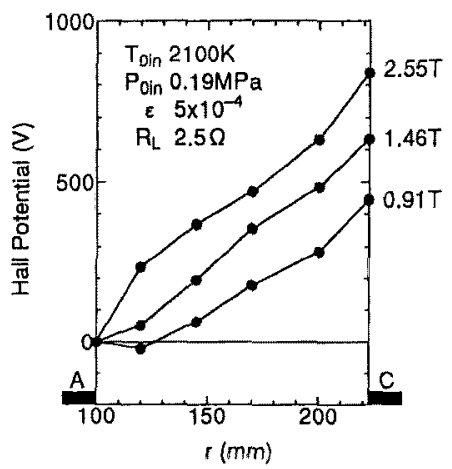

(c) vs. magnetic flux density

Figure 3 Measured Hall potential distributions

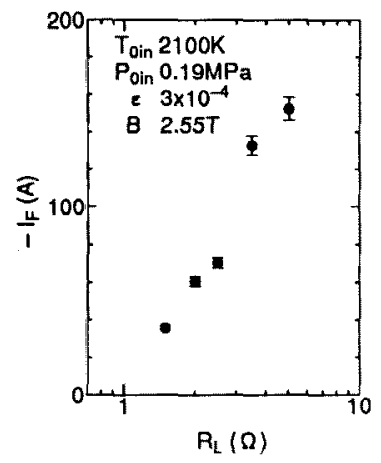

(a) vs. external load resistance

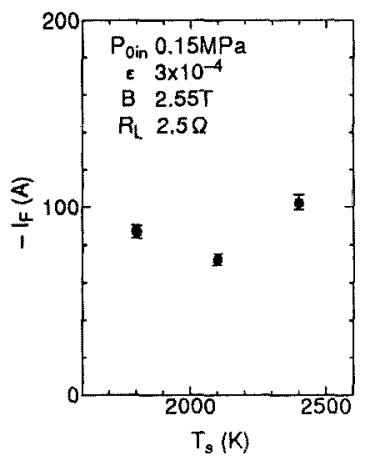

(b) vs. stagnation gas temperature

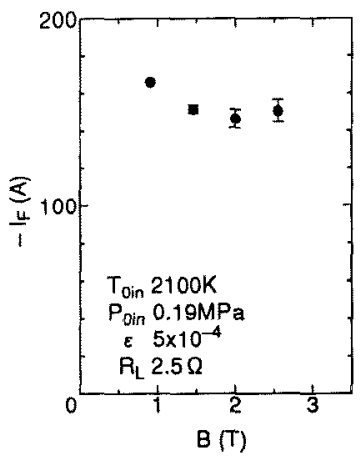

(c) vs. magnetic flux density

Figure 4 Measured Faraday current

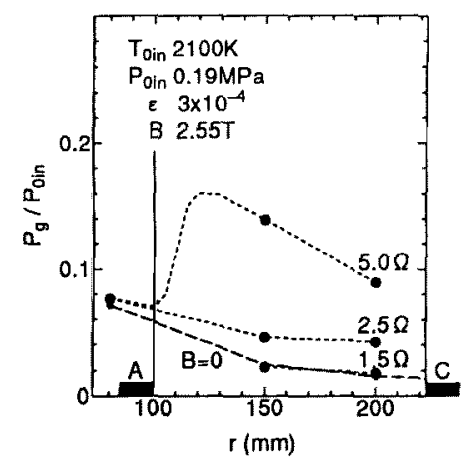

(a) vs. external load resistance

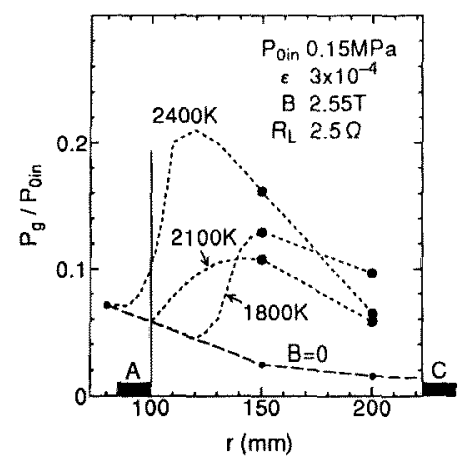

(b) vs. stagnation gas temperature

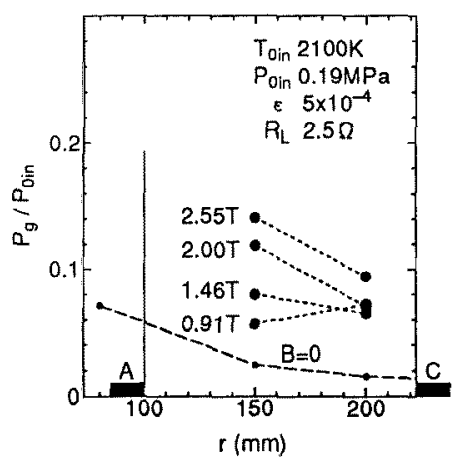

(c) vs. magnetic flux density

Figure 5 Measured static pressure distributions (dotted lines denote the static pressure distribution supposed by the experimental data)

\section{Estimation of Joule Dissipation}

Joule dissipation in the disk generator is estimated on the basis of the above experimental results.

Joule dissipation J.D. is given by

$$
\text { J.D. }=\frac{\vec{j}^{2}}{\sigma}
$$

where $\vec{j}$ is current density vector, $\sigma$ is electrical conductivity.
The Joule dissipation J.D.* is estimated in the present study from the following equation using the measured values,

$$
J . D .^{*}=\frac{\left\langle j_{r}\right\rangle^{2}+\left\langle j_{\theta}\right\rangle^{2}}{\sigma^{*}}
$$

where $j_{\tau}$ and $j_{\theta}$ are the $r$ and $\theta$ components of current density, respectively. The notation \langle\rangle means the average over space and time. 
The electrical conductivity $\sigma^{*}$ is estimated using the following equations which are derived from the generalized Ohm's law,

$$
\begin{aligned}
\beta^{*} & =\frac{\left\langle j_{0}\right\rangle\left\langle E_{r}\right\rangle+\left\langle j_{\theta}\right\rangle\left\langle u_{\theta}\right\rangle\langle B\rangle+\left\langle j_{r}\right\rangle\left\langle u_{r}\right\rangle\langle B\rangle}{\left\langle j_{r}\right\rangle\left\langle E_{r}\right\rangle+\left\langle j_{r}\right\rangle\left\langle u_{0}\right\rangle\langle B\rangle-\left\langle j_{0}\right\rangle\left\langle u_{r}\right\rangle\langle B\rangle} \cdots \\
\sigma^{*} & =\frac{\left(1+\beta^{* 2}\right)\left\langle j_{r}\right\rangle}{\left\langle E_{r}\right\rangle+\beta^{*}\left\langle u_{r}\right\rangle\langle B\rangle+\left\langle u_{0}\right\rangle\langle B\rangle} \cdots \cdots \cdots \cdots
\end{aligned}
$$

where $E_{r}$ is the radial component of electric field, $B$ is the magnetic flux density perpendicular to the $r-\theta$ plane, $u_{r}$ and $u_{\theta}$ are the $r$ and $\theta$ components of gas velocity, respectively.

The averaged values are evaluated as follows:

$$
\begin{aligned}
& \left\langle j_{r}\right\rangle=\frac{I_{H}}{\left(\frac{\int_{\text {in }}^{o u t} 2 \pi r h_{e f f} d r}{\int_{\text {in }}^{o u t} d r}\right)} \cdots \cdots \cdots \cdots \cdots \cdots \cdots \\
& \left\langle j_{\theta}\right\rangle=\frac{I_{F}}{\int_{\text {in }}^{\text {out }} h d r} . \\
& \left\langle E_{r}\right\rangle=\frac{V_{H I}}{\int_{i n}^{\text {out }} d r} \\
& \langle B\rangle=\frac{\int_{i n}^{\text {out }} B d r}{\int_{i n}^{\text {out }} d r} \\
& \left\langle u_{r}\right\rangle=\frac{\int_{\text {in }}^{\text {out }} u_{r} d r}{\int_{\text {in }}^{\text {out }} d r} \\
& \left\langle u_{\theta}\right\rangle=\frac{\int_{\text {in }}^{\text {out }} u_{\theta} d r}{\int_{\text {in }}^{\text {out }} d r}
\end{aligned}
$$

where $I_{H}$ and $I_{F}$ are the Hall and Faraday current, respectively, $V_{H}$ is the Hall voltage, $r$ is the radial distance, in and out are the inlet and outlet of the generator, respectively, $h$ is the channel height, $h_{\text {eff }}$ is the effective channel height estimated from the static pressure distribution in the case of absence of the applied magnetic field.

The reason for the different height of $h$ and $h_{e f f}$ is the different distribution between Hall and Faraday current in the boundary layer. It was reported that the Hall current decreased in the boundary layer due to the reduction of the gas flow velocity, although the Faraday current did not decrease considerably[9]. This approach is, however, not sufficient to discuss the distribution of fluid properties in the boundary layer because the MHD interaction is not considered in $h_{\text {eff }}$. Detailed investigation will be necessary to discuss the effects of it precisely.

The values of $u_{r}$ and $u_{\theta}$ are estimated from the quasione-dimensional calculation taking account of the measured static pressure distributions. The values obtained by this method are in agreement within an error of $10 \%$ with the measured values from the mutual correlation of cesium line intensity.

It must be noted that the Joule dissipation estimated above is thought to be larger than the real one because the nonuniform distributions of fluid properties along $r$ and $z$ directions are not considered sufficiently. If these nonuniform distributions are taken into account, $\sigma^{*}$ will become larger due to its strong nonlinearity. The values of the Joule dissipation estimated in the present study are, however, expected to give good indications.

Power density P.D. and mechanical work of fluid against the Lorentz force $W_{L}$ are given by

$$
\begin{aligned}
P . D . & =-\vec{j} \cdot \vec{E}=-j_{r} E_{r} \ldots \ldots \ldots \ldots \ldots \ldots \\
W_{L} & =-\vec{u} \cdot(\vec{j} \times \vec{B})=-u_{r} j_{\theta} B+u_{\theta} j_{r} B \ldots \ldots
\end{aligned}
$$

These quantities are estimated using the averaged values:

$$
\begin{aligned}
P . D .^{*} & =-\left\langle j_{r}\right\rangle\left\langle E_{r}\right\rangle \cdots \cdots \cdots \cdots \cdots \\
W_{L}^{*} & =-\left\langle u_{r}\right\rangle\left\langle j_{\theta}\right\rangle\langle B\rangle+\left\langle u_{\theta}\right\rangle\left\langle j_{r}\right\rangle\langle B\rangle
\end{aligned}
$$

The variations of the power density $P . D .^{*}$ and the Joule dissipation J.D.* are shown in Fig. 7. The mechanical work of fluid against the Lorentz force $W_{L}$ is equal to the sum of P.D.* and J.D.*. The maximum isentropic efficiency is attained with the load resistance of $2.5 \Omega$ as shown in Fig. 6 (a). When the load resistance becomes lower than this optimum value, the power density decreases while the Joule dissipation remains almost equal. It is known from a simple theory that the Joule dissipation decreases when the loading factor is low. This contradiction may be caused by the nonuniformity of the plasma in the case of low load resistance. When the load resistance becomes higher than the optimum one, the Joule dissipation increases. It is indicated from the present experiments that the optimum load resistance also gives the maximum electrical efficiency which is the ratio of the power density and the mechanical work of fluid.

The Joule dissipation increases when the stagnation temperature is lowered. This is thought to be caused by the low ionization degree at the inlet of the generator.

When the applied magnetic flux density increases, the power density increases remarkably in spite of the slight increase of the Joule dissipation. It is indicated that the higher magnetic flux density result in the higher electrical efficiency as expected by a simple theory.

\section{Mechanisms of Total Pressure Losses}

Two reasons are considered for the reduction of the outlet total gas pressure. One of them is a enthalpy reduction due to the electrical power extraction and a heat transfer loss to the walls. The other is a entropy production due to the Joule dissipation, a wall friction loss and two or three dimensional gasdynamical effects (e.g. shock wave).

If the polytropic efficiency $\eta_{p}$ has a constant value in the generator and the heat transfer loss is neglected, the relation between enthalpy extraction ratio $E . E$. and the reduction of total pressure is written as follows: 


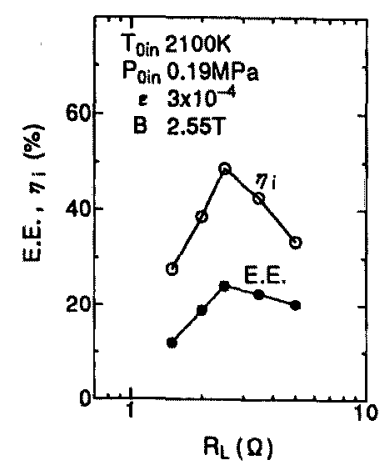

(a) vs. external load resistance

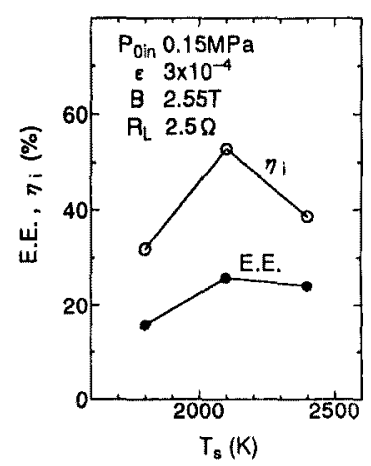

(b) vs. stagnation gas temperature

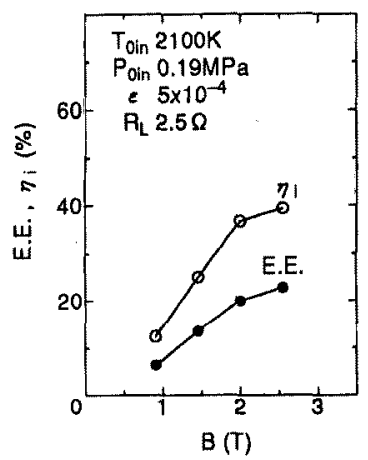

(c) vs. magnetic flux density

Figure 6 The variations of enthalpy extraction ratio and isentropic efficiency

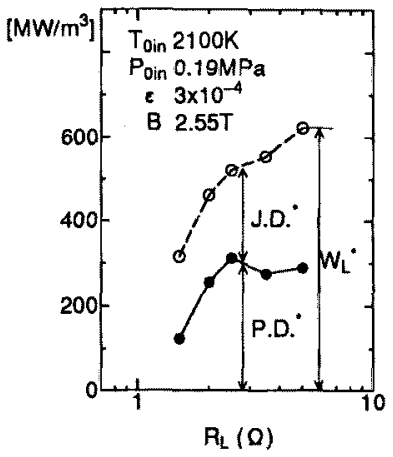

(a) vs. external load resistance

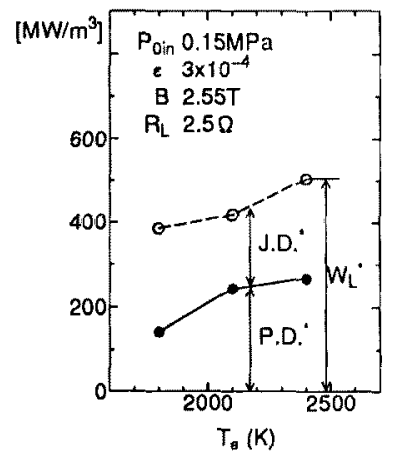

(b) vs. stagnation gas temperature

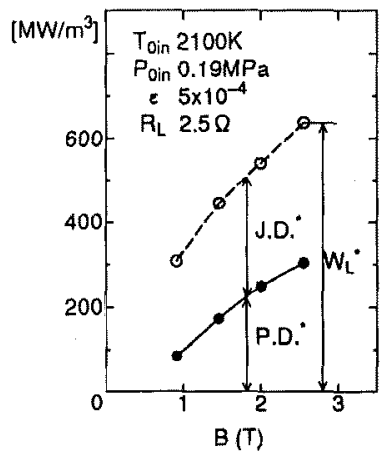

(c) vs. magnetic flux density

J.D. : Joule dissipation estimated from Eq.3

P.D. : power density estimaled from Eq.14

$W_{L}^{*}$ : mechanical work of fluid estimated from Eq.15

Figure 7 Estimated Joule dissipation and power density

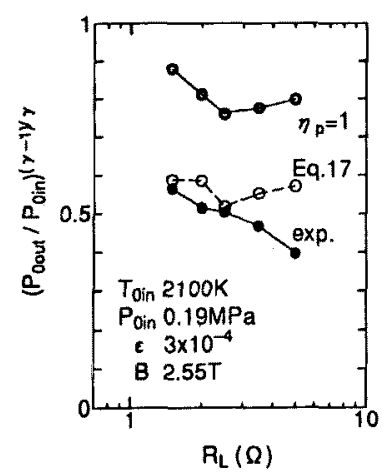

(a) vs. external load resistance

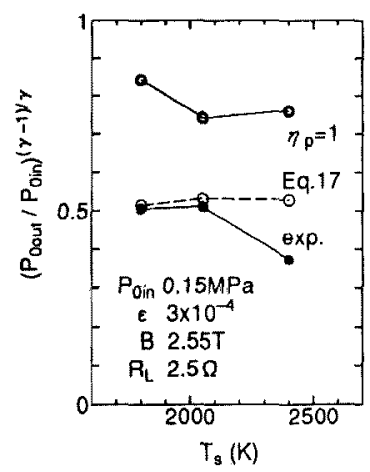

(b) vs. stagnation gas temperature

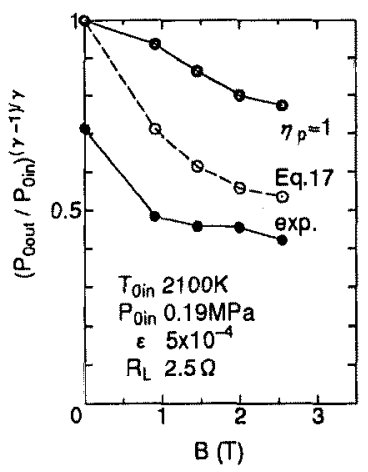

(c) vs. magnetic flux density

- $\quad \eta_{p}=1: \eta_{p}$ is assumed to be 1

- Eq.17: $\eta_{p}$ is estimated from Eq.17

- exp. : obtained from experiment

Figure 8 The variations of total pressure losses 


$$
\left(\frac{p_{0 \text { out }}}{p_{0 \text { in }}}\right)^{\frac{\gamma-1}{\gamma}}=(1-E . E .)^{\frac{1}{\eta_{p}}} \ldots \ldots \ldots \ldots \ldots . .
$$

The polytropic efficiency $\eta_{p}$, which takes account of the Joule dissipation as a entropy production, is written as follows[10]

$$
\eta_{p}=\frac{\eta_{e}}{1+\frac{\gamma-1}{2} M^{2}\left(1-\eta_{e}\right)} .
$$

where $\eta_{e}$ is the electrical efficiency, $M$ is the Mach number of the working gas.

The value of $p_{0 o u t}$ is estimated from Eq. (16) and (17) using the above mentioned electrical efficiency $\left(P . D .{ }^{*} / W_{L}^{*}\right)$ and $\langle M\rangle .\langle M\rangle$ is estimated from the following equations:

$$
\begin{aligned}
\langle M\rangle & \simeq \frac{\langle u\rangle}{\sqrt{\gamma R\left\langle T_{g}\right\rangle}} \cdots \cdots \\
\langle u\rangle & =\left\langle\sqrt{u_{r}^{2}+u_{\theta}^{2}}\right\rangle \cdots \\
\left\langle T_{g}\right\rangle & \simeq \frac{\left\langle T_{0}\right\rangle}{1-\frac{\gamma-1}{2}\langle M\rangle^{2}} \cdots \\
\left\langle T_{0}\right\rangle & \simeq T_{0 i n}\left(1-\frac{E . E .}{2}\right)
\end{aligned}
$$

where $u$ is the gas flow velocity, $T_{g}$ and $T_{0}$ are the static and stagnation temperature, respectively, $T_{0 i n}$ is the inlet stagnation temperature.

The normalized outlet total pressure $\left(p_{0 \text { out }} / p_{0 \text { in }}\right)^{\frac{\gamma-1}{\gamma}}$ is shown in Fig. 8. The mark of $\bullet$ in this Figure denotes the outlet total pressure when the value of $\eta_{p}$ in Eq. (16) is equal to 1 . This value shows the outlet total pressure when the electrical power is extracted isentropically. The mark of $\circ$ denotes the outlet total pressure when the polytropic efficiency is estimated from Eq. (17). The mark of $\bullet$ denotes the measured outlet total pressure.

It should be noticed that the outlet total pressure estimated from Eq. (17), which is denoted by the mark of 0 , tends to be lower than the real one because the Joule dissipation is thought to be overestimated as declared above.

The difference between the values of $\bullet$ and $\circ$ in Fig. 8 means the pressure loss caused by the Joule dissipation. The difference between the values of $\circ$ and - means the pressure loss caused by other entropy production (e.g. wall friction loss, shock wave). The heat transfer loss is neglected here. The value of $(1-0)$ is equal to E.E. which is lead from Eq. (16). The value of $(1-0) /(1-\bullet)$ means the isentropic efficiency, as known from Eq. (1).

With an increase in the load resistance, a decrease in the value of $\bullet$ is found while the value of $\circ$ does not decrease obviously. This suggests an existence of a large entropy production excepting the Joule dissipation (e.g. shock wave due to large Lorentz force) when the value of load resistance is high. This entropy production is thought to be caused by the sudden increase in static pressure at the inlet of the generator.
When the stagnation temperature is high $(2400 \mathrm{~K})$, the difference between the value of 0 and - becomes large. This fact can be explained in the same way as the case of high load resistance.

The difference between the values of $\circ$ and $\bullet$ becomes small with the increase in the magnetic flux density. This indicates that the wall friction loss decreases owing to the decrease in the gas flow velocity. Fig. 8 (c) also shows that the total pressure loss caused by the entropy production, which is the difference between the values of $\bullet$ and $\bullet$, becomes small with the increase in the magnetic flux density, although the enthalpy extraction increases remarkably. The reason for the suppression of the entropy production is both the decrease in the wall friction loss and no remarkable increase in the Joule dissipation. It is indicated from this fact that the higher magnetic flux density, which causes the higher MHD interaction, does not result in the deterioration of the performance of the generator. It is considered that the higher magnetic flux density is essential to get higher isentropic efficiency.

\section{Conclusion}

1. The Joule dissipation in a nonequilibrium MHD disk generator was successfully estimated from empirical approaches. Experimental results showed that the isentropic efficiency of the generator was strongly affected by the value of the Joule dissipation.

2. The reduction of the total pressure caused by the Joule dissipation was estimated. It was suggested that the large entropy production excepting the Joule dissipation existed when a sudden increase in the static pressure appeared at the inlet of the generator.

3. When the applied magnetic flux density increased, the total pressure loss caused by the entropy production was suppressed although the extraction of electrical power increased remarkably. The high MHD interaction caused by the high magnetic flux density did not deteriorate the performance of the generator. The higher magnetic flux density is considered to be essential to get higher isentropic efficiency.

\section{Acknowledgment}

Authors wish to express their great thanks to $\mathrm{Mr}$. M.Matsuda for his contribution to preparation and operation of the facility and data analysis.

(Manuscript received April 19, 1996, revised August 22, 1996)

\section{References}

(1) N. Harada, et al., "Experimental Studies of Closed Cycle MHD Power Generation with FUJI-1 Blow-Down Facility," Proc. of 11st Int. Conf. on MHD, Vol.4, pp.1153-1160, 1992 
(2) H. Yamasaki, et al., "Highest Enthalpy Extraction from Disk MHD Generator with Cesium Seeded Argon," Proc. of 32nd SEAM, 1994

(3) N. Harada, et al., "Demonstration of Enthalpy Extraction Over $30 \%$ with Shock-Tube Driven Disk MHD Generator," Proc. of 31th SEAM, pp.III.2.1-III.2.9, 1993

(4) T. Okamura, et al., "Review and New Results of High Enthalpy Extraction Experiments at Tokyo Institute of Technology," Proc. of 32nd SEAM, 1994

(5) H. Nakamura, et al., "Measurements of Properties Concerning Isentropic Efficiency in a Nonequilibrium MHD Disk Generator," IEEE Trans. on Plasma Science, Vol.24, No.3, 1996

(6) I. Hayashi and Y. Nakano, J.IEE Japan, Vol.81, pp.1084-1092, 1961

(7) T. Okamura, et al., "Measurement of Faraday Current in Disk Generator," Proc. of 16th Symp. of Efficient Use of Energy and Direct Electrical Power Generation, 1994

(8) S.H. Chue, Prog. Aerospace Sci., 16-2, 147, 1975

(9) D. Biswas, et al., "Boundary Layer Effects in He and Ar Driven Disk MHD Generators," Energy Convers. Mgmt Vol.28, No.1, pp.105-115, 1988

(10) R.J. Rosa, "Magnetohydrodynamic Energy Conversion," McGraw-Hill, 1968

Iajime Nakamura (Student member) was born on January 1,1965 . He received his B.E. and M.E. degrees from Tokyo Institute of Technology in 1987 and 1989, respectively. He entered the Nomura Research Institute as a researcher in 1989. He has been a doctor course student in Tokyo Institute of Technology since 1994. His research field is closed cycle disk MHD power generation.

Tetsuji Okamura (Nonmember) was born on August 5,

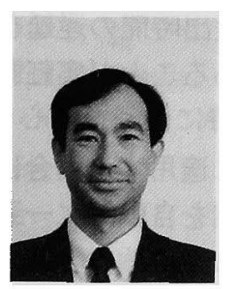
1959. He received his B.E., M.E. and D.E. degrees from Tokyo Institute of Technology in 1982, 1984 and 1987, respectively. He has been an Associate Professor in the Department of Energy Sciences since 1994 at Tokyo Institute of Technology. His work was mainly concentrated on superconducting magnet and closed cycle MHD power generation.
Susumu Shioda

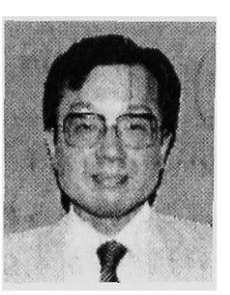

(Member) was born on November 19, 1936. He received his B.S. degree from Tokyo University in 1962 . He entered the Toshiba cooperation as a researcher in 1962. He was a Professor in the Department of Energy Sciences in 1978, and has been a Professor in Department of Environmental Physics and Engineering since 1993 at Tokyo institute of Technology. His work was mainly concentrated on closed cycle MHD power generation. 